\title{
Univalent Functions Defined by a Generalized Multiplier Differential Operator
}

\section{Adnan Ghazy Alamoush}

Faculty of Science, Taibah University, Saudi Arabia

\begin{abstract}
In this paper, we investigate a new subclass of univalent functions defined by a generalized differential operator, and obtain some interesting properties of functions belonging to the class $R_{\lambda, \mu, \alpha, \beta, \gamma, \vartheta}^{m}(\varpi)$.
\end{abstract}

\section{Introduction}

Let $A$ denote the class of the functions $f$ of the form

$$
f(z)=z+\sum_{k=2}^{\infty} a_{k} z^{k}
$$

which are analytic in the open unit disc $\mathbb{U}=\{z \in \mathbb{C}:|z|<1\}$. Let $H(\mathbb{U})$ be the space of holomorphic functions in $\mathbb{U}$. By $S$ and $K$ we denote the subclasses of functions in $A$ which are univalent and convex in $\mathbb{U}$, respectively. Let $P$ be the well-known Carathéodory class of normalized functions with positive real part in $\mathbb{U}$.

The Hadamard product or convolution of the functions

$$
f(z)=z+\sum_{k=2}^{\infty} a_{k} z^{k} \quad \text { and } \quad g(z)=z+\sum_{k=2}^{\infty} b_{k} z^{k}
$$

Received: March 5, 2019; Accepted: April 2, 2019

2010 Mathematics Subject Classification: 30C45.

Keywords and phrases: analytic functions, univalent function, differential operator, differential subordination, convex functions, structural formula.

Copyright (C) 2019 Adnan Ghazy Alamoush. This is an open access article distributed under the Creative Commons Attribution License, which permits unrestricted use, distribution, and reproduction in any medium, provided the original work is properly cited. 
is given by

$$
(f * g)(z)=z+\sum_{k=2}^{\infty} a_{k} b_{k} z^{k}, \quad(z \in \mathbb{U})
$$

We now define a new generalized multiplier differential operator.

Definition 1.1. Let $m \in \mathbb{N}_{0}=\mathbb{N} \cup\{0\}, \quad \alpha, \beta, \lambda, \mu, \rho \geq 0, \quad 0 \leq \gamma \leq \lambda, \quad 0<\varphi \leq 1$, $\alpha+\beta>0$. Then for $f \in A$, we define a new generalized multiplier operator $D_{\lambda, \mu}^{m}(\alpha, \beta, \gamma, \vartheta)$ by

$$
\begin{gathered}
D_{\lambda, \mu}^{0}(\alpha, \beta, \gamma, \vartheta) f(z)=f(z), \\
D_{\lambda, \mu}^{1}(\alpha, \beta, \gamma, \vartheta) f(z) \\
=\frac{[\alpha+\beta+\gamma-(2 \vartheta-1)(\lambda+\mu)] f(z)+[(2 \vartheta-1)(\lambda+\mu)-\gamma] z f^{\prime}(z)+\gamma \lambda z^{2} f^{\prime \prime}(z)}{\alpha+\beta}, \\
\ldots, \\
D_{\lambda, \mu}^{m}(\alpha, \beta, \gamma, \vartheta) f(z)=D_{\lambda, \mu}(\alpha, \beta, \gamma, \vartheta)\left(D_{\lambda, \mu}^{m-1}(\alpha, \beta, \gamma, \vartheta)\right) .
\end{gathered}
$$

Remark 1. If $f(z)$ is given by (1), then from Definition 1.1, we obtain

$$
D_{\lambda, \mu}^{m}(\alpha, \beta, \gamma, \vartheta) f(z)=z+\sum_{k=2}^{\infty} \Omega_{\lambda, \mu}^{m}(\alpha, \beta, \gamma, \vartheta) a_{k} z^{k}
$$

where

$$
\Omega_{\lambda, \mu}^{m}(\alpha, \beta, \gamma, \vartheta)=\left[\frac{\alpha+[(2 \vartheta-1)(\lambda+\mu)+\gamma(k \lambda-1)](k-1)+\beta}{\alpha+\beta}\right]^{m}
$$

From (2) it follows that $D_{\lambda, \mu}^{m}(\alpha, \beta, \gamma, \vartheta) f(z)$ can be written in terms of convolution as

$$
D_{\lambda, \mu}^{m}(\alpha, \beta, \gamma, \vartheta) f(z)=(f * g)(z)
$$


where

$$
g(z)=z+\sum_{k=2}^{\infty} \Omega_{\lambda, \mu}^{m}(\alpha, \beta, \gamma, \vartheta) a_{k} z^{k}
$$

The differential operator $D_{\lambda, \mu}^{m}(\alpha, \beta, \gamma, \vartheta)$ includes many earlier differential operators (see also [2]), which are mentioned below:

- $\quad D_{1,0}^{m}(0,1,0,1):=D^{m} f(z)$, has been studied by Sălăgean [24].

- $\quad D_{1,0}^{m}(1,1,0,1):=L^{m} f(z)$, has been studied by Uralegaddi and Somanatha [26].

- $\quad D_{1,0}^{m}(\alpha, 1,0,1):=L_{\beta}^{m}$, has been studied by Cho and Srivastava [9].

- $\quad D_{\lambda, 0}^{\rho}(0,1,0,1):=D_{\lambda}^{\rho} f(z)$, has been studied by Acu and Owa [1].

- $\quad D_{\lambda, 0}^{m}(0,1,0,1):=D_{\lambda}^{m} f(z)$, has been studied by Al-Oboudi [4].

- $\quad D_{\lambda, 0}^{\rho}(1, \beta, \gamma, 1):=L_{1}(\rho, \lambda, \beta) f(z)$, has been studied by Cătaş et al. [8].

- $\quad D_{\lambda, 0}^{m}(\alpha, 0,0,1):=D_{\lambda}^{m}(\alpha)$, has been studied by Aouf et al. [5].

- $\quad D_{\lambda, 0}^{m}\left(0,1,0, \frac{\alpha+\beta}{2}\right):=D_{\alpha, \beta, 0, \lambda}^{m} f(z)$, has been studied Alamoush and Darus [3].

- $\quad D_{\lambda, 0}^{m}(0,1, \gamma, 1):=D_{\lambda, \gamma}^{m} f(z)$, has been studied by Răducanu and Orhan [23] (see also [20]).

- $\quad D_{\lambda, \mu}^{m}(\alpha, \beta, 0,1):=D_{\lambda}^{m}(\alpha, \beta, \mu)$, has been studied by Darus and Faisal [11].

- $\quad D_{\lambda, \mu}^{m}(\alpha, 0,0,1):=D_{\lambda}^{m}(\alpha, \mu)$, has been studied by Darus and Faisal [10].

Definition 1.2. Let $m \in \mathbb{N}_{0}=\mathbb{N} \cup\{0\}, \quad \varpi \in[0,1), \quad \alpha, \beta, \lambda, \mu \geq 0, \quad 0 \leq \gamma \leq \lambda$, $0<\varphi \leq 1, \quad \alpha+\beta>0$. Then a function $f \in A$ is said to be in the class $R_{\lambda, \mu, \alpha, \beta, \gamma, \vartheta}^{m}(\varpi)$, if it satisfies the condition 


$$
\Re\left[D_{\lambda, \mu}^{m}(\alpha, \beta, \gamma, \vartheta)\right]^{\prime}>\Phi, \quad z \in \mathbb{U}
$$

Remark 2. It is clear that the following classes

1. $R_{\lambda, 0,0,1,0,1}^{0}(\varpi) \equiv R_{\lambda}^{0}(\varpi) \equiv R(\varpi) \equiv R_{0}^{m}(\varpi)$ and that $R_{\lambda}^{1}(\varpi) \equiv R_{\lambda}(\varpi)$, the class of functions $f \in A$ satisfying $\Re\left[f^{\prime}(z)+\lambda z f^{\prime \prime}(z)\right]>\Phi, z \in \mathbb{U}$ studied by Ponnusamy [22] and other.

2. $R_{1,0,0,1,0,1}^{m}(\varpi) \equiv R^{m}(\varpi) \equiv M_{m}(\varpi)$, the class of functions $f \in A$ satisfying $\Re\left[D^{m} f(z)\right]^{\prime}>\Phi, \quad z \in \mathbb{U}$ studied by Oros [21].

3. $R_{\lambda, 0,0,1,0,1}^{m}(\varpi) \equiv R_{\lambda}^{m}(\varpi)$, the class of functions $f \in A$ satisfying $\Re\left[D_{\lambda}^{m} f(z)\right]^{\prime}$ $>\varpi, \quad z \in \mathbb{U}$ studied by Al-Oboudi [4].

4. $R_{\lambda, 0,0,1, \gamma, 1}^{m}(\varpi) \equiv R_{\lambda, \gamma}^{m}(\varpi)$, the class of functions $f \in A$ satisfying $\Re\left[D_{\lambda, \gamma}^{m} f(z)\right]^{\prime}$ $>\Phi, \quad z \in \mathbb{U}$ studied by Zhou and Xu [17].

The main object of this paper is to present a systematic investigation for the class $R_{\lambda, \mu, \alpha, \beta, \gamma, \vartheta}^{m}(\varpi)$. In particular, for this function class, we derive an inclusion result, structural formula, extreme points, coefficient bound, convolution property and other interesting properties.

\section{Preliminaries}

In order to prove our results, we will make use of the following lemmas.

Lemma 2.1. [18] Let $h \in K$, and let $A \geq 0$. Suppose that $B(z)$ and $D(z)$ are analytic in $\mathbb{U}$, with $D(0)=0$ and

$$
\mathfrak{R}(B(z)) \geq A+4\left|\frac{D(z)}{h^{\prime}(0)}\right|, \quad z \in \mathbb{U} .
$$

If an analytic function $p$ with $p(0)=h(0)$ satisfies

$$
A z^{2} p^{\prime \prime}(z)+B(z) z p^{\prime}(z)+p(z)+D(z) \prec h(z), \quad z \in \mathbb{U},
$$


then

$$
p(z) \prec h(z), \quad z \in \mathbb{U} \text {. }
$$

Lemma 2.2. [19] Let $q$ be a convex function in $\mathbb{U}$ and let

$$
h(z)=q(z)+\varpi_{z} q^{\prime}(z)
$$

where $\varpi>0$. If $p \in H(\mathbb{U})$ with

$$
p(z)=q(0)+p_{1} z+p_{2} z^{2}+\cdots \quad \text { and } \quad p(z)+\varpi_{z p^{\prime}}(z) \prec h(z), \quad z \in \mathbb{U},
$$

then

$$
p(z) \prec q(z), \quad z \in \mathbb{U},
$$

and this result is sharp.

Lemma 2.3. [25] If $p(z)$ is analytic in $\mathbb{U}, p(0)=1$ and, $\mathfrak{R}(P(z))>\frac{1}{2}, z \in \mathbb{U}$, then for any function $F$ analytic in $\mathbb{U}$, the function $P * F$ takes values in the convex hull of the image of $\mathbb{U}$ under $F$.

Note that the symbol “ $\prec$ ” stands for subordination throughout this paper.

3. Coefficient Bounds for the Function Class $B_{\Sigma}^{k, \alpha, \beta, \delta, \lambda}(\gamma, \phi)$

Theorem 3.1. Let $m \in \mathbb{N}_{0}=\mathbb{N} \cup\{0\}, \quad \varpi \in[0,1), \quad \alpha, \beta, \lambda, \mu \geq 0, \quad 0 \leq \gamma \leq \lambda$, $0<\varphi \leq 1, \alpha+\beta>0$. Then $R_{\lambda, \mu, \alpha, \beta, \gamma, \vartheta}^{m+1}(\varpi) \subset R_{\lambda, \mu, \alpha, \beta, \gamma, \vartheta}^{m}(\varpi)$.

Proof. Let $f \in R_{\lambda, \mu, \alpha, \beta, \gamma, \vartheta}^{m+1}(\varpi)$. By using the properties of the operator $R_{\lambda, \mu, \alpha, \beta, \gamma, \vartheta}^{m}(\varpi)$, we get

$$
\begin{aligned}
& D_{\lambda, \mu}^{m+1}(\alpha, \beta, \gamma, \vartheta) f(z) \\
& {[\alpha+\beta+\gamma-(2 \vartheta-1)(\lambda+\mu)] D_{\lambda, \mu}^{m}(\alpha, \beta, \gamma, \vartheta) f(z)} \\
& =\frac{+[(2 \vartheta-1)(\lambda+\mu)-\gamma] z\left(D_{\lambda, \mu}^{m}(\alpha, \beta, \gamma, \vartheta) f(z)\right)^{\prime}}{\alpha+\beta}
\end{aligned}
$$




$$
+\frac{\gamma \lambda z^{2}\left(D_{\lambda, \mu}^{m}(\alpha, \beta, \gamma, \vartheta) f(z)\right)^{\prime \prime}}{\alpha+\beta}
$$

Differentiating (6) with respect to $z$, we obtain

$$
\left(D_{\lambda, \mu}^{m+1}(\alpha, \beta, \gamma, \vartheta) f(z)\right)^{\prime}=p(z)+\left[\frac{(2 \vartheta-1)(\lambda+\mu)+2 \lambda \gamma}{\alpha+\beta}\right] p^{\prime}(z)+\left[\frac{\gamma \lambda}{\alpha+\beta}\right] p^{\prime \prime}(z)
$$

where

$$
p(z)=\left(D_{\lambda, \mu}^{m}(\alpha, \beta, \gamma, \vartheta) f(z)\right)^{\prime}
$$

Since $f \in R_{\lambda, \mu, \alpha, \beta, \gamma, \vartheta}^{m+1}(\varpi)$, by using Definition 1.2 and (7), we have

$$
\Re\left\{p(z)+\left[\frac{(2 \vartheta-1)(\lambda+\mu)+2 \lambda \gamma}{\alpha+\beta}\right] p^{\prime}(z)+\left[\frac{\gamma \lambda}{\alpha+\beta}\right] p^{\prime \prime}(z)\right\}>\Phi, \quad z \in \mathbb{U},
$$

which is equivalent to

$$
\left\{p(z)+\left[\frac{(2 \vartheta-1)(\lambda+\mu)+2 \lambda \gamma}{\alpha+\beta}\right] p^{\prime}(z)+\left[\frac{\gamma \lambda}{\alpha+\beta}\right] p^{\prime \prime}(z)\right\} \prec \frac{1+(2 \Phi-1) z}{1-z} \equiv h(z) .
$$

From Lemma 2.1, with $A=\frac{\gamma \lambda}{\alpha+\beta}, B(z)=\frac{(2 \vartheta-1)(\lambda+\mu)+2 \lambda \gamma}{\alpha+\beta}$, and $D(z)=0$, we have $p(z) \prec h(z)$, which implies that $\mathfrak{R}\left\{\left(D_{\lambda, \mu}^{m}(\alpha, \beta, \gamma, \vartheta) f(z)\right)^{\prime}\right\}>\Phi, z \in \mathbb{U}$. Hence $f \in R_{\lambda, \mu, \alpha, \beta, \gamma, \vartheta}^{m}(\varpi)$ and the proof of the theorem is complete.

Clearly $\quad R_{\lambda, \mu, \alpha, \beta, \gamma, \vartheta}^{m}(\varpi) \subset R_{\lambda, \mu, \alpha, \beta, \gamma, \vartheta}^{m-1}(\varpi) \subset \cdots \subset R_{\lambda, \mu, \alpha, \beta, \gamma, \vartheta}^{0}(\varpi) \subset S \quad$ (see $[12,14])$.

Now, we will show that the set $R_{\lambda, \mu, \alpha, \beta, \gamma, \vartheta}^{m}(\varpi)$ is convex (see [17]).

Theorem 3.2. The set $R_{\lambda, \mu, \alpha, \beta, \gamma, \vartheta}^{m}(\varpi)$ is convex.

Proof. Let the functions

$$
f_{i}(z)=z+\sum_{k=2}^{\infty} a_{i} z^{k} \quad(i=1,2)
$$


be in the class $R_{\lambda, \mu, \alpha, \beta, \gamma, \vartheta}^{m}(\varpi)$. It is sufficient to show that the function $h(z)=$ $\chi_{1} f_{1}(z)+\chi_{2} f_{2}(z)$, with $\chi_{1}$ and $\chi_{2}$ nonnegative and $\chi_{1}+\chi_{2}=1$, is in the class $R_{\lambda, \mu, \alpha, \beta, \gamma, \vartheta}^{m}(\varpi)$.

Since

$$
h(z)=z+\sum_{k=2}^{\infty}\left(\chi_{1} a_{k_{1}}+\chi_{2} a_{k_{2}}\right) z^{k}
$$

then we have

$$
\left[D_{\lambda, \mu}^{m}(\alpha, \beta, \gamma, \vartheta) h(z)\right]^{\prime}=1+\sum_{k=2}^{\infty} k\left(\chi_{1} a_{k_{1}}+\chi_{2} a_{k_{2}}\right)\left[\varpi_{\lambda, \mu}^{m}(\alpha, \beta, \gamma, \vartheta)\right] z^{k-1}
$$

hence

$$
\begin{aligned}
& \Re\left(D_{\lambda, \mu}^{m}(\alpha, \beta, \gamma, \vartheta) h(z)\right)^{\prime} \\
= & \Re\left(1+\chi_{1} \sum_{k=2}^{\infty} k\left[\varpi_{\lambda, \mu}^{m}(\alpha, \beta, \gamma, \vartheta)\right] a_{k_{1}} z^{k-1}\right) \\
& +\Re\left(1+\chi_{2} \sum_{k=2}^{\infty} k\left[\varpi_{\lambda, \mu}^{m}(\alpha, \beta, \gamma, \vartheta)\right] a_{k_{2}} z^{k-1}\right)-1 .
\end{aligned}
$$

Since $f_{1}, f_{2} \in R_{\lambda, \mu, \alpha, \beta, \gamma, \vartheta}^{m}(\varpi)$, this implies that

$$
\Re\left(1+\chi_{i} \sum_{k=2}^{\infty} k\left[\varpi_{\lambda, \mu}^{m}(\alpha, \beta, \gamma, \vartheta)\right] a_{k_{i}} z^{k-1}\right)>1+\chi_{i}(\varpi-1) .
$$

Using (9) in (8), we obtain

$$
\Re\left(D_{\lambda, \mu}^{m}(\alpha, \beta, \gamma, \vartheta) h(z)\right)^{\prime}>1+\varpi\left(\chi_{1}+\chi_{2}\right)-\left(\chi_{1}+\chi_{2}\right)
$$

and since $\chi_{1}+\chi_{2}=1$, the theorem is proved.

Theorem 3.3. Let $q$ be convex function with $q(0)=1$ and let $h$ be a function of the form $h(z)=q(z)+z q^{\prime}(z), \quad z \in \mathbb{U}$. If $f \in A$ satisfies the differential subordination $\left(D_{\lambda, \mu}^{m}(\alpha, \beta, \gamma, \vartheta)\right)^{\prime} \prec h(z), \quad z \in \mathbb{U}$, then $D_{\lambda, \mu}^{m}(\alpha, \beta, \gamma, \vartheta) / z \prec q(z)$ and the result is sharp. 
Proof. If we let $p(z)=D_{\lambda, \mu}^{m}(\alpha, \beta, \gamma, \vartheta) / z, z \in \mathbb{U}$, then we get $\left(D_{\lambda, \mu}^{m}(\alpha, \beta, \gamma, \vartheta)\right)^{\prime}$ $=p(z)+z p^{\prime}(z)$. So the subordination $\left(D_{\lambda, \mu}^{m}(\alpha, \beta, \gamma, \vartheta)\right)^{\prime} \prec h(z), \quad z \in \mathbb{U}$, becomes $p(z)+z p^{\prime}(z)=q(z)+z q^{\prime}(z), z \in \mathbb{U}$. Hence from Lemma 2.2, we have $\left(D_{\lambda, \mu}^{m}(\alpha, \beta, \gamma, \vartheta)\right) / z \prec q(z)$. The result is sharp.

\section{Structural Formula}

In this section, a structural formula, extreme points and coefficient bounds for functions in $R_{\lambda, \mu, \alpha, \beta, \gamma, \vartheta}^{m}(\varpi)$ are obtained.

Theorem 4.1. A function $f \in A$ is in the class $R_{\lambda, \mu, \alpha, \beta, \gamma, \vartheta}^{m}(\varpi)$ if and only if it can be expressed as

$$
f(z)=\left[z+\sum_{k=2}^{\infty} \frac{1}{\Omega_{\lambda, \mu}^{m}(\alpha, \beta, \gamma, \vartheta)} z^{k}\right] * \int_{|\zeta|=1}\left[z+2(1-\bar{\sigma}) \bar{\zeta} \sum_{k=2}^{\infty} \frac{(\zeta z)^{k}}{k}\right] d \sigma(\zeta)
$$

where $\Omega_{\lambda, \mu}^{m}(\alpha, \beta, \gamma, \vartheta)$ is given by (3) and $\sigma$ is a positive probability measure defined on the unit circle $\mathbb{T}=\{\zeta \in \mathbb{C}:|\zeta|<1\}$.

Proof. From (4) it follows that, $f \in R_{\lambda, \mu, \alpha, \beta, \gamma, \vartheta}^{m}(\varpi)$ if and only if

$$
\frac{\left(D_{\lambda, \mu}^{m}(\alpha, \beta, \gamma, \vartheta) f(z)\right)^{\prime}-\varpi}{1-\varpi} \in P
$$

Using Herglotz integral representation of functions in Carathéodory class $P$ (see [13] and [15]), there exists a positive Borel probability measure $\sigma$ such that

$$
\frac{\left(D_{\lambda, \mu}^{m}(\alpha, \beta, \gamma, \vartheta) f(z)\right)^{\prime}-\Phi}{1-\Phi}=\int_{|\zeta|=1} \frac{1+\zeta z}{1-\zeta z} d \sigma(\zeta), \quad z \in \mathbb{U}
$$

which is equivalent to

$$
\left(D_{\lambda, \mu}^{m}(\alpha, \beta, \gamma, \vartheta) f(z)\right)^{\prime}=\int_{|\zeta|=1} \frac{1+(1-2 \Phi) \zeta z}{1-\zeta z} d \sigma(\zeta), \quad z \in \mathbb{U} .
$$


Integrating (11), we obtain

$$
\begin{aligned}
D_{\lambda, \mu}^{m}(\alpha, \beta, \gamma, \vartheta) f(z) & =\int_{0}^{z}\left[\int_{|\zeta|=1} \frac{1+(1-2 \Phi) \zeta u}{1-\zeta u} d \sigma(\zeta)\right] d u \\
& =\int_{|\zeta|=1}\left[\int_{0}^{z} \frac{1+(1-2 \Phi) \zeta u}{1-\zeta u} d u\right] d \sigma(\zeta)
\end{aligned}
$$

that is

$$
D_{\lambda, \mu}^{m}(\alpha, \beta, \gamma, \vartheta)=\int_{|\zeta|=1}\left[z+2(1-\varpi) \bar{\zeta} \sum_{k=2}^{\infty} \frac{(\zeta z)^{k}}{k}\right] d \sigma(\zeta)
$$

Equality (10) follows now, from (4), (5) and (12). Since the converse of this deductive process is also true, we have proved our theorem.

Corollary 4.2. The extreme points of the class $R_{\lambda, \mu, \alpha, \beta, \gamma, \vartheta}^{m}(\varpi)$ are

$$
f(z)_{\zeta}=z+2(1-\varpi) \bar{\zeta} \sum_{k=2}^{\infty} \frac{(\zeta z)^{k}}{k \Omega_{\lambda, \mu}^{m}(\alpha, \beta, \gamma, \vartheta)}, \quad z \in \mathbb{U},|\zeta|=1
$$

Proof. Consider the functions

$$
g_{\zeta}(z)=z+2(1-\varpi) \bar{\zeta} \sum_{k=2}^{\infty} \frac{(\zeta z)^{k}}{k} \quad \text { and } \quad g_{\sigma}(z)=\int_{|\zeta|=1} g_{\zeta}(z) d \sigma(\zeta)
$$

Since the map $\sigma \rightarrow g_{\zeta}$ is one-to-one, making use of (5), (6) and (12), the assertion follows from (10) (see [7]).

From Corollary 4.3, we can obtain coefficient bounds for the functions in the class $R_{\lambda, \mu, \alpha, \beta, \gamma, \vartheta}^{m}(\varpi)$.

Corollary 4.3. If $f \in R_{\lambda, \mu, \alpha, \beta, \gamma, \vartheta}^{m}(\varpi)$ is given by $(1)$, then

$$
\left|a_{k}\right| \leq \frac{2(1-\varpi)}{k \Omega_{\lambda, \mu}^{m}(\alpha, \beta, \gamma, \vartheta)}, \quad k \geq 2
$$

The result is sharp. 
The coefficient bounds are maximized at an extreme point. Therefore, the result follows from (13).

Corollary 4.4. If $f \in R_{\lambda, \mu, \alpha, \beta, \gamma, \vartheta}^{m}(\varpi)$, then, for $|z|=r<1$

$$
r-2(1-\varpi) r^{2} \sum_{k=2}^{\infty} \frac{1}{k \Omega_{\lambda, \mu}^{m}(\alpha, \beta, \gamma, \vartheta)} \leq|f(z)| \leq r+2(1-\varpi) r^{2} \sum_{k=2}^{\infty} \frac{1}{k \Omega_{\lambda, \mu}^{m}(\alpha, \beta, \gamma, \vartheta)}
$$

and

$$
1-2(1-\varpi) r \sum_{k=2}^{\infty} \frac{1}{k \Omega_{\lambda, \mu}^{m}(\alpha, \beta, \gamma, \vartheta)} \leq|f(z)| \leq 1+2(1-\varpi) r \sum_{k=2}^{\infty} \frac{1}{k \Omega_{\lambda, \mu}^{m}(\alpha, \beta, \gamma, \vartheta)}
$$

\section{Convolution Property}

In this part, we prove the analogue of the Pólya-Schoenberg conjecture for the class $R_{\lambda, \mu, \alpha, \beta, \gamma, \vartheta}^{m}(\varpi)$.

Theorem 5.1. The class $R_{\lambda, \mu, \alpha, \beta, \gamma, \vartheta}^{m}(\Phi)$ is closed under the convolution with a convex function. That is, if $f \in R_{\lambda, \mu, \alpha, \beta, \gamma, \vartheta}^{m}(\varpi)$ and $g \in C$, then $f * g \in$ $R_{\lambda, \mu, \alpha, \beta, \gamma, \vartheta}^{m}(\varpi)$.

It is known that if $g$ is convex univalent in $U$, then (see [18])

$$
\Re\left\{\frac{g(z)}{z}\right\}>\frac{1}{2} \text {. }
$$

Using convolution properties, we have

$$
\Re\left(D_{\lambda, \mu}^{m}(\alpha, \beta, \gamma, \vartheta)(f * g)(z)\right)^{\prime}=\mathfrak{R}\left(\left[D_{\lambda, \mu}^{m}(\alpha, \beta, \gamma, \vartheta)(f)\right]^{\prime} * \frac{g(z)}{z}\right)
$$

and the result follows by application of Lemma 2.3.

Corollary 5.2. The class $R_{\lambda, \mu, \alpha, \beta, \gamma, \vartheta}^{m}(\varpi)$ is invariant under Bernardi integral operator.

Proof. Let $R_{\lambda, \mu, \alpha, \beta, \gamma, \vartheta}^{m}(\varpi)$. The Bernardi integral operator is defined as (see [6]): 


$$
F_{c}(f)(z)=\frac{1+c}{z^{k}} \int_{0}^{z} t^{c-1} f(t) d t, \quad(c \in A, c>-1) .
$$

It is easy to check that $F_{c}(f)(z)=(f * g)(z)$, where

$$
g(z)=\sum_{k=1}^{\infty} \frac{1+c}{k+c} z^{k}=\frac{1+c}{z^{k}} \int_{0}^{z} \frac{t^{c}}{1-t} f(t) d t, \quad(z \in \mathbb{U}, c>-1)
$$

Since the function $\phi(z)=\frac{z}{1-z}, z \in \mathbb{U}$ is convex, it follows (see [16]) that the function $g$ is also convex. From Theorem 5.1, we obtain $F_{c}(f) \in R_{\lambda, \mu, \alpha, \beta, \gamma, \vartheta}^{m}(\varpi)$. Therefore, $F_{c}\left[R_{\lambda, \mu, \alpha, \beta, \gamma, \vartheta}^{m}(\varpi)\right] \subset R_{\lambda, \mu, \alpha, \beta, \gamma, \vartheta}^{m}(\varpi)$.

\section{Conclusion}

In this article, by using the Hadamard product or convolution, we define a new generalized multiplier operator. Also, we have presented new subclass of univalent functions and we have investigated some geometric properties like inclusion result, structural formula, extreme points, coefficient bound and convolution property. By Varying the parameter in results, several well-known results have been obtained by Ponnusamy [22], Oros [21], Al-Oboudi [4], and Zhou and Xu [17] as shown in above Remark 2.

\section{References}

[1] M. Acu and S. Owa, Note on a Class of Starlike Functions, RIMS, Kyoto (2006), 1-10.

[2] A. G. Alamoush and M. Darus, On certain subclasses of analytic functions defined by generalized differential operators, ROMAI J. 11 (2015), 17-31.

[3] A. Alamoush and M. Darus, New criteria for certain classes containing generalised differential operator, J. Qual. Measure. Anal. 9 (2013), 59-71.

[4] F. M. Al-Oboudi, On univalent functions defined by a generalized Sălăgean operator, Int. J. Math. Math. Sci. (2004), no. 25-28, 1429-1436.

[5] M. K. Aouf, R. M. El-Ashwah and S. M. El-Deeb, Some inequalities for certain $p$-valent functions involving extended multiplier transformations, Proc. Pakistan Acad. Sci. 46 (2009), 217-221. 
[6] S. D. Bernardi, Convex and starlike functions, Trans. Amer. Math. Soc. 135 (1969), 429446.

[7] L. Brickman, T. H. MacGregor and D. R. Wilken, Convex hulls of some classical families of univalent functions, Trans. Amer. Math. Soc. 156 (1971), 91-107.

[8] A. Cătaş, G. I. Oros and G. Oros, Differential subordinations associated with multiplier transformations, Abstr. Appl. Anal. (2008), Art. ID 845724, 1-11.

[9] N. E. Cho and H. M. Srivastava, Argument estimates of certain analytic functions defined by a class of multiplier transformations, Math. Comput. Modelling 37 (2003), 39-49.

[10] M. Darus and I. Faisal, Characterization properties for a class of analytic functions defined by generalized Cho and Srivastava operator, Proc. 2nd Inter. Conf. Math. Sci. (2010), 1106-1113.

[11] M. Darus and I. Faisal, Some subclasses of analytic functions defined by generalized differential operator, Acta Univ. Apulensis 29 (2012), 197-215.

[12] A. W. Goodman, Univalent Functions, Tampa, FL: Mariner Publishing Company Inc., 1984.

[13] D. J. Hallenbeck and T. H. MacGregor, Linear Problems and Convexity Techniques in Geometric Function Theory, London, UK: Pitman, 1984.

[14] W. Kaplan, Close-to-convex schlicht functions, Michigan Math. J. 1 (1953), 169-185.

[15] W. Keopf, A uniqueness theorem for functions of positive real part, J. Math. Sci. 28 (1994), 78-90.

[16] Z. Lewandowski, S. S. Miller and E. Zlotkiewicz, Generating functions for some classes of univalent functions, Proc. Amer. Math. Soc. 56 (1976), 111-117.

[17] Li Zhou and Qing-hua Xu, On univalent functions defined by the multiplier differential operator, Int. J. Math. Anal. 6 (2012), 735-742.

[18] S. S. Miller and P. T. Mocanu, Differential Subordinations: Theory and Applications, New York, NY: Marcel-Dekker, 2000.

[19] S. S. Miller and P. T. Mocanu, On some classes of first-order differential subordinations, Michigan Math. J. 32 (1985), 185-195.

[20] H. Orhan, D. Erhan and Ç. Murat, Fekete-Szegö problem for certain subclasses of analytic functions, Demonstratio Math. 45(4) (2012), 835-846.

[21] G. I. Oros, A class of holomorphic functions defined using a differential operator, Gen. Math. 13(4) (2005), 13-18. 
[22] S. Ponnusamy, Differential subordination and starlike functions, Complex Variables Theory Appl. 19(3) (1992), 185-194.

[23] D. Răducanu and H. Orhan, Subclasses of analytic functions defined by a generalized differential operator, Int. J. Math. Anal. 4 (2010), 1-15.

[24] G. S. Sălăgean, Subclasses of univalent functions, Complex analysis-fifth RomanianFinnish seminar, Part 1 (Bucharest, 1981), Lecture Notes in Math., 1013, Springer, Berlin, 1983, pp. 362-372.

[25] R. Singh and S. Singh, Convolution properties of a class of starlike functions, Proc. Amer. Math. Soc. 106 (1989), 145-152.

[26] B. A. Uralegaddi and C. Somanatha, Certain classes of univalent functions, in: Current Topics in Analytic Function Theory, River Edge, NJ: World Sci. Publ. (1992), 371-374. 